Check for updates

Cite this: Phys. Chem. Chem. Phys., 2020, 22, 28173

Received 28th July 2020,

Accepted 20th November 2020

DOI: $10.1039 / \mathrm{d} 0 \mathrm{cp} 04012 \mathrm{f}$

rsc.li/pccp

\title{
Viscosity effects on optically generated electron and nuclear spin hyperpolarization $\dagger$
}

\author{
Matthew W. Dale, (D) ${ }^{a}$ Daniel J. Cheney, (D) ${ }^{b}$ Claudio Vallotto (iD ${ }^{a}$ and \\ Christopher J. Wedge (D)*ab
}

\begin{abstract}
Spin hyperpolarization can dramatically increase signal intensities in magnetic resonance experiments, providing either improved bulk sensitivity or additional spectroscopic detail through selective enhancements. While typical hyperpolarization approaches have utilized microwave irradiation, one emerging route is the use of optically generated triplet states. We report an investigation into the effects of solution viscosity on radical-triplet pair interactions, propose a new standard for quantification of the hyperpolarization in EPR experiments, and demonstrate a significant increase in the optically generated ${ }^{1} \mathrm{H}$ NMR signal enhancement upon addition of glycerol to aqueous solutions.
\end{abstract}

\section{Introduction}

Nuclear magnetic resonance (NMR) and electron paramagnetic resonance (EPR) offer versatile probes of chemical information which are indispensable to the modern physical scientist. Nevertheless magnetic resonance methods are hampered by low intrinsic sensitivity arising from weak thermal Boltzmann polarization of the relevant nuclear or electronic spin energy levels. ${ }^{1}$ Population differences across the relevant spectroscopic transitions are therefore small, such that only a tiny fraction of the spins are actually observed, e.g. only 1 in $10^{5}$ for ${ }^{1} \mathrm{H}$ at $14.1 \mathrm{~T}$ (600 MHz NMR). A range of hyperpolarization methods, providing greater population differences and hence higher sensitivity than attainable with thermal spin systems, are now under investigation. ${ }^{1,2}$ We recently demonstrated that photochemically generated electronic hyperpolarization can be transferred to nuclei to enhance sensitivity in solution-state $\mathrm{NMR}^{3}{ }^{3}$ a method we refer to as optical DNP (dynamic nuclear polarization). Here we examine how optical generation of hyperpolarization in the electron spin-system can be maximised through changes to the solution environment, achieving an increased optical NMR enhancement and providing insights relevant to direct use of electronic hyperpolarization in EPR spectroscopy. ${ }^{4-8}$

Optically generated triplet states often have a large initial electron spin polarization which has been utilized directly in both EPR and NMR studies. ${ }^{9}$ In the solid-state application of

\footnotetext{
${ }^{a}$ Department of Physics, University of Warwick, Gibbet Hill Road, Coventry, CV4 $7 A L, U K$

${ }^{b}$ Department of Chemical Sciences, University of Huddersfield, Huddersfield, HD1 3DH, UK. E-mail: c.wedge@hud.ac.uk

$\dagger$ Electronic supplementary information (ESI) available: Further experimental details, simulation parameters and additional data. See DOI: 10.1039/d0cp04012f
}

triplet polarization to enhance sensitivity in EPR distance measurements using pulsed dipolar spectroscopy have recently attracted significant interest, ${ }^{10-13}$ whereas in NMR impressive enhancements have been observed for matrix nuclei but are much reduced upon transfer to a solution-state substrate. ${ }^{14,15}$ In solution although some triplet states can still be relatively long-lived they undergo rapid spin relaxation, hence their EPR spectra are not normally observed and direct exploitation of their initial polarization is difficult. It is however possible for state mixing in the encounter pair formed by a radical and unpolarized triplet to lead to electronic hyperpolarization of a radical. ${ }^{16}$ The distinguishing feature of this radical triplet pair mechanism $(\mathrm{RTPM})^{17}$ as compared to other spin chemical mechanisms generating hyperpolarized electron spin states, such as the radical pair, relaxation and triplet mechanisms, ${ }^{9,18}$ is that hyperpolarization can be generated on a persistent rather than transient radical species. The RTPM therefore has great potential to provide hyperpolarization that can be utilized more generally for sensitivity enhancement, without the complication of radical reaction or decay.

In the RTPM photoexcitation of a suitable dye molecule followed by rapid intersystem crossing generates an excited triplet state which undergoes collisional encounters with a stable doublet radical. During these encounters the energy levels of the combined radical-triplet pair are best described as quartet and doublet states, which are separated by the electron exchange interaction $(J)$, Fig. 1. The doublet levels are rapidly depopulated by spin-allowed transitions to the ground state of the dye molecule making the radical an efficient triplet quencher by the so-called enhanced intersystem crossing (EISC) mechanism. ${ }^{19}$ Due to spin-state mixing driven by the zero-field splitting of the triplet molecule this process results in generation of net polarization of the radical, which is emissive in the case of a positive exchange interaction. ${ }^{18}$ 

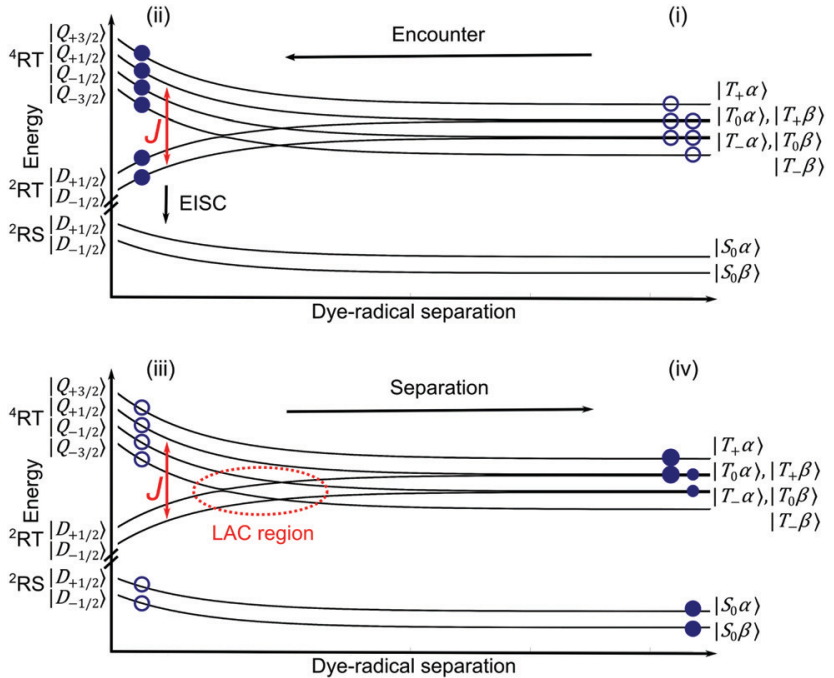

Fig. 1 Schematic energy level diagram of a radical and dye molecule. (i) At large separations the spin states of the two molecules are independent. Shortly after photoexcitation triplet sub-levels $\left(T_{+}, T_{0}, T_{-}\right)$of the dye are equally populated with only a small Boltzmann polarization difference (not visible) of the radical levels ( $\alpha$ and $\beta$ ). (ii) Upon diffusive encounter the resultant radical-triplet complex occupies exchange split quartet and doublet states. Spin-allowed quenching by the process known as enhanced intersystem crossing (EISC) selectively depopulates the doublet states to produce radical-singlet pair character (iii). Finally the pair separates, with passage through the level anti-crossing (LAC) region resulting in generation of emissive electron spin polarization of the radical (iv), with overpopulation of the $\left|T_{+} \alpha\right\rangle$ and $\left|T_{0} \alpha\right\rangle$ states. ${ }^{18}$ The diagram assumes a constant applied magnetic field resulting in equal Zeeman splitting of the radical and triplet energy levels $\left(g_{\mathrm{R}} \approx g_{\mathrm{T}} \approx 2\right)$.

The majority of early studies of the RTPM focussed on polyaromatic triplet sensitizers in organic solvents, but recently the electron spin polarization efficiency of the RTPM was investigated for a range of xanthene dyes and nitroxide radicals in aqueous solution. ${ }^{20}$ Not only is generation of hyperpolarization in aqueous solution essential to applications in biological systems, but unusually large electronic polarizations were observed in these systems; in the case of Rose Bengal and 2,2,6,6-tetramethylpiperidine-1-oxyl (TEMPO) the net electron spin polarization $\left(P_{\text {net }}\right)$ generated by each radical triplet encounter was reported to be $\mathbf{- 1 5 0}$ times the thermal electron spin polarization $\left(P_{\mathrm{eq}}\right) \cdot{ }^{20}$ This large polarization was attributed to slow rotational and translational diffusion of the dianionic Rose Bengal molecule at the neutral pH used. Having already demonstrated transfer of this large electronic hyperpolarization to nuclei, ${ }^{3}$ in the present study the scope to further enhance the polarization level through increasing the solvent viscosity is considered, and the effects on both hyperpolarized EPR and NMR spectra are reported.

\section{Experimental}

All experiments reported were carried out at room temperature $(\sim 295 \mathrm{~K})$. Key experimental details are given below with further information available in the ESI. $\dagger$

\section{EPR spectroscopy}

Pulsed and continuous-wave (CW) EPR spectra were recorded using a Bruker Elexsys E580 spectrometer with a dielectric resonator (Bruker EN 4118X-MD4). All pulsed EPR experiments were echo detected using a standard $90^{\circ}-\tau-180^{\circ}-\tau$-echo sequence, with $\tau=120 \mathrm{~ns}$ and the receiver protection time reduced to $230 \mathrm{~ns}$ in order to allow detection of the fast relaxing nitroxide radicals. Relaxation time $T_{1 \mathrm{e}}$ was measured using an inversion recovery sequence. Due to the high dielectric loss of the aqueous solvent mixture the sample volume was reduced by using $1.1 \mathrm{~mm}$ inner diameter quartz EPR tubes (Wilmad WG221).

Samples were illuminated in the EPR resonator using a frequency-doubled Nd:YAG (Continuum Surelite SLI-20 Hz) whose beam diameter of $6 \mathrm{~mm}$ filled the optical window of the resonator. For synchronisation with the microwave pulse sequence the laser was operated in DAT mode with a fixed Q-switch delay set using a digital delay generator (Quantum Composer QC9512) triggered by the EPR spectrometer. Adjustment of laser power was achieved using a Glan-laser polarizer and half-wave plate (Thorlabs GL10A and WPMH05M-532) in rotation mounts. Laser power levels were checked using a volume absorbing head (Gentec UP19K-15S-VR).

\section{NMR spectroscopy}

NMR spectra were recorded using the radiofrequency coil of a Bruker X-band ENDOR resonator (EN 4118X-MD4) which was tuned to $14.6 \mathrm{MHz}$ and matched using an external circuit (Bruker H132224). Data were acquired using a Bruker Avance spectrometer, and the external magnetic field of $0.342 \mathrm{~T}$ was supplied by a 10" EPR electromagnet (Bruker ER 073) with $12 \mathrm{~kW}$ power supply. Considerations of field stability and homogeneity when using this arrangement with a resistive magnet have been discussed previously, along with the construction of our $1 \mathrm{~mm}$ internal diameter quartz flow cell. ${ }^{3}$ The spectra recorded are unreferenced, with chemical shift reported relative to the $\mathrm{H}_{2} \mathrm{O}$ solvent peak.

Samples were illuminated using a diode-pumped solid-state laser at $532 \mathrm{~nm}$ (MGL-F-532-2W), gated by an optical shutter (Lasermet LS-10) controlled by a TTL pulse from the NMR console. A pair of N-BK7 lenses were used to expand the beam diameter before a multi-order half-wave plate in rotation mount and polarizing beamsplitter (Thorlabs WPMH05M-532 and CCM1-PBS251/M) used to adjust laser power. This was measured using a surface absorbing thermal sensor (Thorlabs S310C). A cylindrical lens was used to fill the optical window of the resonator.

\section{Sample flow}

In order to avoid photodegradation of samples both EPR and NMR measurements were performed using a flow system operating at $10 \mu \mathrm{lmin}^{-1}$ driven by a syringe pump (Legato 110). Deoxygenation was achieved by bubbling dry nitrogen gas through the sample contained in a gas tight glass syringe (SGE 50MR-LL-GT) for $20 \mathrm{~min}$ prior to the experiment. To prevent oxygen ingress during the experiment and also reduce laser 
induced sample heating, nitrogen gas flowed through an outer jacket around the PTFE sample tubing as described previously. ${ }^{3}$

\section{Results and discussion}

\section{Time-resolved EPR data}

The time-evolution of electron spin-polarization was monitored by pulsed EPR spectroscopy by altering the delay between the triplet generating laser pulse and an electron spin echo sequence. Compared to direct transient EPR detection (continuous microwaves with no field modulation $)^{21}$ this delay after flash experiment has the benefit of detecting the thermal magnetization $\left(S_{0}\right)$, thereby allowing quantification of the spin-polarization effect. A similar approach with detection of the free-induction decay has been used before, ${ }^{20}$ but we found the echo detection experiment to be preferable owing to compatibility with a 4-step phase cycle which removes artefacts arising from the effect of the laser pulse on the EPR resonator. Data obtained for a range of volume fractions of glycerol are shown in Fig. 2a, from which it can be seen that the peak magnetization reaches nearly 13 times the thermal equilibrium signal.

All triplets may be considered to form instantaneously on the EPR timescale as intersystem crossing from the singlet to triplet excited state of the dye takes places on a picosecond timescale, and the laser pulse width of approximately $5 \mathrm{~ns}$ is comparable to normal microwave EPR pulse widths. The peak emissive signal intensity is reached rapidly as the triplets are quenched by radicals which become spin-polarized, followed by a slower decay to thermal equilibrium governed by electron spin lattice relaxation (timescale $T_{1 \mathrm{e}}$ ). Addition of glycerol notably alters the time profile, lowering the peak magnetization obtained but also significantly extending the lifetime of the polarization.
Molecular oxygen as a ground state triplet is an effective quencher of the triplet state of the dye molecule, competing with quenching by the nitroxide radicals. As shown in Fig. S1a (ESI $\dagger$ ) the removal of oxygen from the sample therefore significantly increases the peak magnitude of the magnetization detected. The decay kinetics are also slower in the deoxygenated case as a result of (i) an increase in the effective triplet lifetime $1 / k_{\mathrm{d}}$ and (ii) an increase in the electronic relaxation time $T_{1 \mathrm{e}}$ of approximately $50 \%$ in the absence of the Heisenberg exchange contribution from dissolved oxygen (values in ESI $\dagger$ ). Sample deoxygenation by nitrogen bubbling was therefore carried out in all cases as described in the Experimental section. This is in contrast to previous work on the Rose Bengal/TEMPO system, and along with the fact that we chose not to use a pH buffer (see below), results in a larger and longer lived magnetization in Fig. 2a than reported in ref. 20 .

In order to extract information on the efficiency of generation of electron spin-polarization the time evolution of the magnetization may be modelled using a kinetic approach based on the Bloch equations..$^{20,22,23}$ While previous work used a simple kinetic scheme we have found that excellent agreement between simulations and experiment can be obtained by using a more detailed photochemical reaction scheme arising from a recent comprehensive study of the photochemistry of Rose Bengal in water. ${ }^{24}$ The full scheme is as follows:

$$
\begin{gathered}
{ }^{1} \mathrm{D}^{2-} \stackrel{h \nu}{\longrightarrow}{ }^{1} \mathrm{D}^{2-\star} \rightarrow{ }^{3} \mathrm{D}^{2-\star} \\
{ }^{3} \mathrm{D}^{2-\star} \stackrel{k_{\mathrm{d}}}{\longrightarrow}{ }^{1} \mathrm{D}^{2-} \\
{ }^{3} \mathrm{D}^{2-\star}+{ }^{3} \mathrm{D}^{2-\star} \stackrel{k_{\mathrm{tt}}}{\longrightarrow}{ }^{1} \mathrm{D}^{2-}+{ }^{1} \mathrm{D}^{2-} \\
{ }^{3} \mathrm{D}^{2-\star}+{ }^{2} \mathrm{R} \stackrel{k_{\mathrm{q}}}{\longrightarrow}{ }^{1} \mathrm{D}^{2-}+{ }^{2} \mathrm{R}^{\#}
\end{gathered}
$$
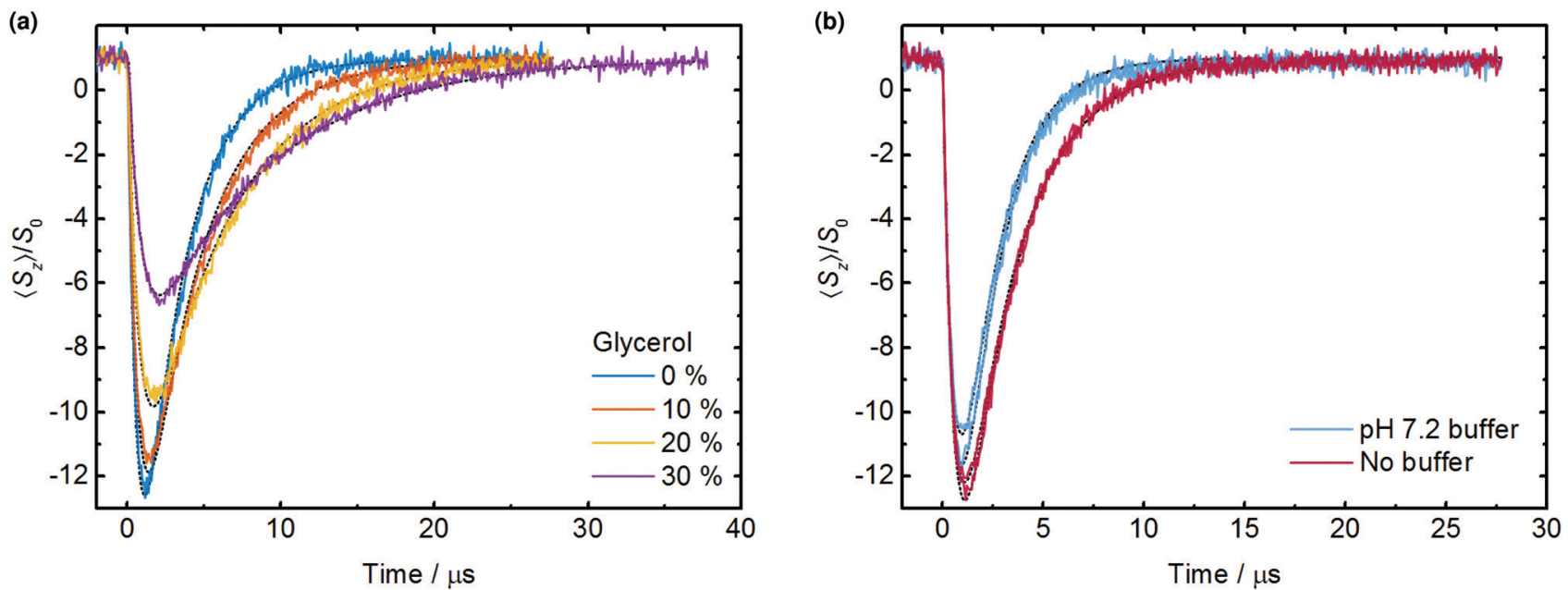

Fig. 2 Delay after flash EPR experiments for (a) solutions with varying amounts of glycerol in the solvent with percentage by volume as indicated (unbuffered) and (b) water solutions with and without pH 7.2 phosphate buffer (two replicates of each condition). Sample composition 0.2 mM Rose Bengal, $0.2 \mathrm{mM}$ TEMPO, deoxygenated by $\mathrm{N}_{2}$ bubbling; illumination $6 \mathrm{~mJ}$ per pulse at $532 \mathrm{~nm}$. Dashed lines are fits to the data. As described in the text, triplet concentration was determined based on the buffered solution for which $P_{\text {net }} / P_{\text {eq }}=-150$ has been reported, ${ }^{20}$ and was held constant in the other fits to permit the relative value of $P_{\text {net }} / P_{\text {eq }}$ to be determined. The uncertainty in the determined fit parameters is provided in the main text and Table 1 . 


$$
\begin{aligned}
& { }^{2} \mathbf{R}^{\# \stackrel{1 / T_{1 \mathrm{e}}}{\longrightarrow}}{ }^{2} \mathbf{R} \\
& { }^{3} \mathrm{D}^{2-\star}+{ }^{1} \mathrm{D}^{2-} \stackrel{k_{\mathrm{sq}}}{\longrightarrow}{ }^{1} \mathrm{D}^{2-}+{ }^{1} \mathrm{D}^{2-} \\
& { }^{3} \mathrm{D}^{2-\star}+{ }^{1} \mathrm{D}^{2-} \stackrel{k_{\text {redox }}}{\longrightarrow}{ }^{1} \mathrm{D}^{\bullet-}+{ }^{1} \mathrm{D}^{\bullet 3-} \\
& { }^{3} \mathrm{D}^{2-\star}+{ }^{3} \mathrm{D}^{2-\star} \stackrel{k_{\text {redox }} \star}{\longrightarrow}{ }^{1} \mathrm{D}^{\bullet-}+{ }^{1} \mathrm{D}^{\bullet 3-} \\
& { }^{1} \mathrm{D}^{\bullet-}+{ }^{1} \mathrm{D}^{\bullet 3-} \stackrel{k_{\text {bet }}}{\longrightarrow}{ }^{1} \mathrm{D}^{2-}+{ }^{1} \mathrm{D}^{2-}
\end{aligned}
$$

where $\mathrm{D}^{2-}$ and $\mathrm{R}$ represent the dianionic Rose Bengal dye molecule and neutral radical respectively, ${ }^{3} \mathrm{D}^{2-\star}$ denotes the triplet excited state and rates are indicated above the reaction arrows. Eqn (1)-(5) are those considered in previous timeresolved EPR studies, ${ }^{20}$ eqn (1) representing triplet formation governed by the triplet quantum yield, and loss processes given by; eqn (2) non-radiative decay and eqn (3) triplet-triplet annihilation. Eqn (4) shows the crucial quenching of the triplet dye by a radical to produce a spin-hyperpolarized radical denoted as ${ }^{2} R^{\#}$. This radical relaxes to thermal equilibrium according to the timescale $T_{1 \mathrm{e}}$ of electron spin-lattice relaxation, eqn (5). Additional processes relating to the dye are; eqn (6) self-quenching, eqn (7) electron exchange with ground state dye or eqn (8) disproportionation with another triplet to give reduced ${ }^{1} \mathrm{D}^{\bullet-3-}$ and oxidised dye ${ }^{1} \mathrm{D}^{\bullet-}$. Eqn (9) represents back electron transfer of these redox forms to regenerate the starting dianionic dye. This scheme leads to the following coupled differential equations:

$$
\begin{aligned}
& \frac{\mathrm{d}\left\langle S_{\mathrm{Z}}\right\rangle}{\mathrm{d} t}=-\frac{\left\langle S_{\mathrm{Z}}\right\rangle-P_{\mathrm{eq}}\left[{ }^{2} \mathrm{R}\right]}{T_{1 \mathrm{e}}}+P_{\text {net }} k_{\mathrm{q}}\left[{ }^{2} \mathrm{R}\right]\left[{ }^{3} \mathrm{D}^{2-\star}\right] \\
& \frac{\mathrm{d}\left[{ }^{3} \mathrm{D}^{2-\star}\right]}{\mathrm{d} t}=-k_{\mathrm{d}}\left[{ }^{3} \mathrm{D}^{2-\star}\right]-k_{\mathrm{q}}\left[{ }^{2} \mathrm{R}\right]\left[{ }^{3} \mathrm{D}^{2-\star}\right] \\
& -\left(k_{\mathrm{sq}}+k_{\text {redox }^{\star}}\right)\left[{ }^{1} \mathrm{D}^{2-}\right]\left[{ }^{3} \mathrm{D}^{2-\star}\right] \\
& -2\left(k_{\mathrm{tt}}+k_{\text {redox }}{ }\right)\left[{ }^{3} \mathrm{D}^{2-\star}\right]^{2} \\
& \frac{\mathrm{d}\left[{ }^{1} \mathrm{D}^{2-}\right]}{\mathrm{d} t}=k_{\mathrm{d}}\left[{ }^{3} \mathrm{D}^{2-\star}\right]+\left(k_{\mathrm{sq}}-k_{\text {redox }}\right)\left[{ }^{1} \mathrm{D}^{2-}\right]\left[{ }^{3} \mathrm{D}^{2-\star}\right] \\
& +2 k_{\mathrm{tt}}\left[{ }^{3} \mathrm{D}^{2-\star}\right]^{2}+2 k_{\mathrm{bet}}\left[{ }^{1} \mathrm{D}^{\bullet-3-}\right]\left[{ }^{1} \mathrm{D}^{\bullet-}\right] \\
& \frac{\mathrm{d}\left[{ }^{1} \mathrm{D}^{\bullet-}\right]}{\mathrm{d} t}=\frac{\mathrm{d}\left[{ }^{1} \mathrm{D}^{\bullet-3}\right]}{\mathrm{d} t}=k_{\text {redox }}{ }^{\star}\left[{ }^{1} \mathrm{D}^{2-}\right]\left[{ }^{3} \mathrm{D}^{2-\star}\right] \\
& +k_{\text {redox } \star \star}\left[{ }^{3} \mathrm{D}^{2-\star}\right]^{2}-k_{\text {bet }}\left[{ }^{1} \mathrm{D}^{\bullet 3-}\right]\left[{ }^{1} \mathrm{D}^{\bullet-}\right]
\end{aligned}
$$

in which $\left\langle S_{\mathrm{Z}}\right\rangle$ is the expectation value of the electronic magnetization arising from the stable radicals (TEMPO), which have an equilibrium electron spin-polarization $P_{\text {eq }}$, and $P_{\text {net }}$ is the polarization generated by each triplet-radical quenching event. Given the already complex nature of the scheme the concentration of polarized radicals $\left({ }^{2} R^{\#}\right)$ was assumed to be small such that the concentration of unpolarized radicals $\left({ }^{2} R\right)$ may be considered constant.
Table 1 Variation in EPR observed RTPM polarization parameters with added glycerol in deoxygenated aqueous solutions of Rose Bengal and TEMPO, both $0.2 \mathrm{mM}$. Polarization values $P_{\text {net }}$ are given relative to the value for a solution with $\mathrm{pH} 7.2$ phosphate buffer and no added glycerol. The uncertainty quoted is the standard deviation of the relative polarization value from fitting across a range of laser powers. Peak values of the transient magnetization $\left(S_{\text {eff }}=\left(S_{0}-\left\langle S_{z}\right\rangle\right) / S_{0}\right)$ are reported for $6 \mathrm{~mJ}$ per pulse illumination at $532 \mathrm{~nm}$. Electron spin-lattice relaxation times were measured before and after each set of polarization experiments with the average and standard deviation given

\begin{tabular}{lccr}
\hline Volume fraction glycerol/\% & $P_{\text {net }}$ (relative) & Max. $s_{\text {eff }}$ & $T_{1 \mathrm{e}} / \mathrm{ns}$ \\
\hline 0 & 1.92 & 13.6 & $620 \pm 20$ \\
10 & $2.4 \pm 0.2$ & 12.9 & $670 \pm 10$ \\
20 & $2.29 \pm 0.09$ & 10.8 & $745 \pm 3$ \\
30 & $1.92 \pm 0.04$ & 7.4 & $807 \pm 2$ \\
\hline
\end{tabular}

The time-profile of the magnetization $\left\langle S_{\mathrm{Z}}(t)\right\rangle$ was simulated by numerically solving eqn (10)-(13) to produce a fit to the experimental data. For standard aqueous solutions the kinetic parameters relating to Rose Bengal were taken from ref. 24 and the electron spin-lattice relaxation rate $T_{1 \mathrm{e}}$ was measured using an inversion recovery pulse sequence (Table 1). With this information the MATLAB ${ }^{25}$ solver ode 45 was used to compute the EPR time profiles, with variable parameters as described below determined by using the lsqcurvefit routine to minimise the sum of squares deviation between the simulated and experimental transients. The kinetic parameters used are given in Table S1 (ESI $\dagger$ ).

If the initial triplet concentration generated by the laser pulse is known the only remaining variable parameter in fitting the experimental data is the polarization ratio $P_{\text {net }} / P_{\text {eq. }}$. It is theoretically possible to calculate triplet concentrations using the laser illumination intensity, extinction coefficient of the dye and quantum yield of triplet formation. A global fit across multiple illumination intensities ought then to produce a unique value for the polarization ratio, ${ }^{20}$ however incomplete and non-uniform illumination of the total sample volume contributing to the EPR signal complicates this approach, and calculated triplet concentrations are prone to errors arising from slight variations in laser focus and unquantified optical losses such as refraction from the curved surface of the capillary sample tube. To avoid these difficulties and as the polarization ratio for the Rose Bengal/TEMPO system has already been determined for an aqueous solution to be $P_{\text {net }} /$ $P_{\text {eq }}=-150$ (ref. 20), this value was taken as a fixed reference and all polarization values are given relative to this system. Delay after flash EPR data for the Rose Bengal/TEMPO system in aqueous solution with the same $\mathrm{pH} 7.2$ phosphate buffer as in this previous work were recorded in duplicate and fitted with the triplet concentration as the sole variable parameter (Fig. 2b), providing a convenient way to determine the initial Rose Bengal triplet concentration as $0.143 \pm 0.005 \mathrm{mM}$ in our experimental arrangement with $532 \mathrm{~nm}$ illumination at $6 \mathrm{~mJ}$ per pulse. When the experiment was repeated under identical conditions but without $\mathrm{pH}$ buffer the polarization was longer lived. A fit to this data can only be obtained by reducing the radical quenching rate $k_{\mathrm{q}}$ from $1.7 \times 10^{9} \mathrm{M}^{-1} \mathrm{~s}^{-1}$ 
to $(8.9 \pm 0.3) \times 10^{8} \mathrm{M}^{-1} \mathrm{~s}^{-1}$, which holding the previously determined triplet concentration constant requires that $P_{\text {net }} / P_{\text {eq }}=$ $-287.6 \pm 0.1$, an increase of 1.92 times relative to the same sample in a buffered solution. The work described below was therefore carried out using deoxygenated aqueous solutions without $\mathrm{pH}$ buffer. The origin of the buffer effect remains unknown but is not thought to be a simple $\mathrm{pH}$ effect, the nitrogen bubbled solutions being at close to neutral $\mathrm{pH}$ (see ESI $\dagger$ ); investigations into $\mathrm{pH}$ effects in these samples are ongoing and will be reported elsewhere.

Data were collected for laser pulse energies from 1 to $6 \mathrm{~mJ}$. In order to fit these time profiles while holding all other parameters constant a non-linear variation in triplet concentration with laser pulse energy was necessary. This implies a non-linear scaling of triplet concentration with photon flux, which may be rationalised by considering the known tendency of Rose Bengal to form intermolecular aggregates at concentrations above $1 \mu \mathrm{M}^{26,27}$

Measurements across the same range of laser powers were repeated for each of the solvent mixtures containing added glycerol, without adjusting the laser alignment. The $6 \mathrm{~mJ}$ per pulse data are shown in Fig. 2a, with full laser power dependent data sets in Fig. S2 (ESI $\dagger$ ). As noted above addition of glycerol to the aqueous solvent leads to a reduction in the peak magnetization and a lengthening of the magnetization decay profile. The prolonged magnetization is a result of a substantial increase of the electron spin-lattice relaxation time with glycerol addition (Table 1). Spin relaxation mechanisms in nitroxides have been studied extensively by Eaton and Eaton, and found to have contributions from spin rotation, modulation of the large $g$-factor and hyperfine anisotropies of the nitroxide, and a thermally activated process. ${ }^{28,29}$ Slower spin relaxation in glycerol containing solutions (up to 69\% added glycerol) was previously shown to arise from an increase in the tumbling correlation time of the radical, which affects both the spin rotation and modulation of $g$ - and hyperfine anisotropies. ${ }^{29}$ While the reproducibility of solid-state DNP studies has been reported to be affected by polymorphism of water-glycerol solutions upon freezing to below $150 \mathrm{~K},{ }^{30}$ to our knowledge there are no reports of polymorphism influencing relaxation properties at ambient temperatures. As our measurements are carried out exclusively at room temperature $(\sim 295 \mathrm{~K})$, over $100 \mathrm{~K}$ above the glass transition temperature of the waterglycerol mixtures used and in a composition region not subject to reported liquid-liquid transitions, ${ }^{31}$ polymorphism is not expected to affect our study. We also note that any clustering of radicals in the water-glycerol mixtures would increase the relaxation rate $\left(T_{1 \mathrm{e}}\right)^{-1}$ of the radicals in the glycerol containing solutions, which is the opposite behaviour to that we observe.

Increasing the volume fraction of glycerol also affects the triplet lifetime by a reduction in the rate of radical quenching $k_{\mathrm{q}}$ and other bimolecular decay processes arising from slower diffusion in the more viscous media. The initial polarization generation from the Rose Bengal triplet therefore occurs over a longer timescale, permitting electron spin relaxation to begin to contribute such that the peak magnetization obtained is reduced. Fits to the data in Fig. S2b-d (ESI $\dagger$ ) are obtained assuming the initial triplet concentration for each laser power to be invariant upon addition of glycerol, and using the increased values of $T_{1 \mathrm{e}}$ measured by inversion recovery. The reduction in the rates of the reaction steps are accounted for by scaling the relevant rate constants by the ratio of viscosities (Table S1, ESI $\dagger$ ). In this way excellent fits to the data are obtained with the only free parameter being the polarization ratio $P_{\text {net }} / P_{\text {eq }}$. The magnitude of the polarization shows a moderate increase upon addition of small amounts of glycerol but at $30 \%$ glycerol by volume is identical within error to the sample without glycerol (Table 1), although the peak polarization is lower and polarization lifetime significantly extended. This illustrates the inadequacy of the polarization ratio in isolation for comparison of the polarization characteristics of different systems.

The increase in $P_{\text {net }}$ with addition of $10 \%$ glycerol is in line with earlier experimental results for the 1-chloronaphthalene $(1 \mathrm{CN}) /$ TEMPO system for which the increase in net polarization with solvent viscosity upon cooling has been extensively studied. $^{32-34}$ This effect has been related to reduced translational diffusion, enabling the radical-triplet pair to spend more time in the crossing region of the quartet and doublet states. This has led to attempts to increase polarization by chemically linking the dye and radical to further restrict motion. ${ }^{35}$ The unusually large dynamic electron polarization of the xanthene dyes has also been attributed to the slow diffusional motion of the strongly hydrated dianionic dye molecules, and slow rotational diffusion highlighted as critical to polarization generation to prevent rotational averaging of the anisotropic zero-field splitting interaction that drives state mixing. ${ }^{20}$

Analytical expressions relating $P_{\text {net }}$ to the relative diffusion coefficient $D_{\mathrm{r}}$ have been derived for the weak and strong exchange limits, ${ }^{34}$ with the strong-exchange result $P_{\text {net }} \propto 1 / D_{\mathrm{r}}$ when $D_{\mathrm{r}} \rightarrow 0$ applicable to xanthene/nitroxide systems at X-band. ${ }^{20}$ Deviations from this simple linear relationship between $P_{\text {net }}$ and viscosity have previously been observed for $1 \mathrm{CN} / \mathrm{TEMPO}$ in highly viscous media $(\eta>20 \mathrm{mPa}$ s). Kobori et al. were able to model the observed viscosity dependence numerically and attributed the deviation to neglected back transitions during slow passage through the level-crossing region. ${ }^{33}$ Shushin revised the earlier analytical expressions to account for Kobori's data resulting in a simple expression of the form $P_{\text {net }} \propto 1 /\left(\right.$ constant $\left.+D_{\mathrm{r}}\right){ }^{36}$ This cannot reproduce the present result of decreasing polarization in the more viscous mixtures, which in fact exhibit $D_{\mathrm{r}}$ values of 2.1 to $6.2 \times 10^{-10} \mathrm{~m}^{2} \mathrm{~s}^{-1}$, spanning the linear dependence range of the earlier work. Shushin's analytical expression has been used to calculate the polarization efficiency of a range of dye and radical systems in benzene and water solutions, but we note experimentally observed variations in $P_{\text {net }}$ between Rose Bengal and Erythrosine-B or between different radicals with Eosin-Y (which we also see in the Rose Bengal case, Fig. S1b, ESI $\dagger$ ) were not reproduced. ${ }^{20}$ At present the range of chemical systems studied experimentally is rather limited with further investigations needed to test the validity of the existing theory and determine 
the reasons for numerous discrepancies when treating xanthene dyes. Such work is beyond the scope of the present study, so having identified that glycerol addition can alter the magnitude and timescale of electron spin-polarization in the Rose Bengal/TEMPO system we now move on to consider the resultant effects on the optically generated nuclear hyperpolarization arising from cross-relaxation of the electronnuclear spin system.

\section{Nuclear polarization}

The ${ }^{1} \mathrm{H}$ NMR signals of the solvent mixtures described above were measured at $14.6 \mathrm{MHz}$ in the presence and absence of illumination from a $532 \mathrm{~nm}$ continuous-wave laser. The nuclear hyperpolarization generated was quantified in the conventional way by the enhancement factor $\varepsilon=\left(\left\langle I_{\mathrm{Z}}\right\rangle-I_{0}\right) / I_{0}$ where $\left\langle I_{\mathrm{Z}}\right\rangle$ is the expectation value of the nuclear polarization with optical pumping and $I_{0}$ the thermal equilibrium nuclear polarization in the abscence of optical pumping. As shown in Fig. 3a the addition of even $10 \%$ glycerol by volume is associated with a significant increase in the NMR enhancement over the pure water ( $0 \%$ glycerol) case. In fact for a fully protonated solvent mixture with $20 \%$ added glycerol (Fig. $3 \mathrm{~b}$ ) the enhancement of $-6.4 \pm 0.4$ exceeds the largest previously reported enhancement of $-3.6 \pm 0.6$ for a deuterated solvent mixture $\left(80 \% \mathrm{D}_{2} \mathrm{O} /\right.$ $20 \% \mathrm{H}_{2} \mathrm{O}$ by volume) with optimized concentrations of $0.2 \mathrm{mM}$ Rose Bengal and 1.0 mM TEMPO. ${ }^{3}$

An upgraded optical system permitted preliminary investigation into optimal illumination conditions. Whereas our earlier study with a relatively divergent $520 \mathrm{~nm}$ laser diode source was power limited, ${ }^{3}$ Fig. 3a shows that with a collimated $532 \mathrm{~nm}$ laser the NMR enhancement of glycerol containing solutions drops for powers over $1.0 \mathrm{~W}$. Given sample flow is used to reduce photodegradation this is likely due to heating effects and shorter illumination periods were therefore trialled to reduce the heating duty cycle. As shown in Fig. 3c the enhancement achieved is increased further by using a higher laser power of $2.0 \mathrm{~W}$ gated to $4 \mathrm{~s}$ of illumination immediately prior to the NMR acquisition which was repeated at a fixed rate
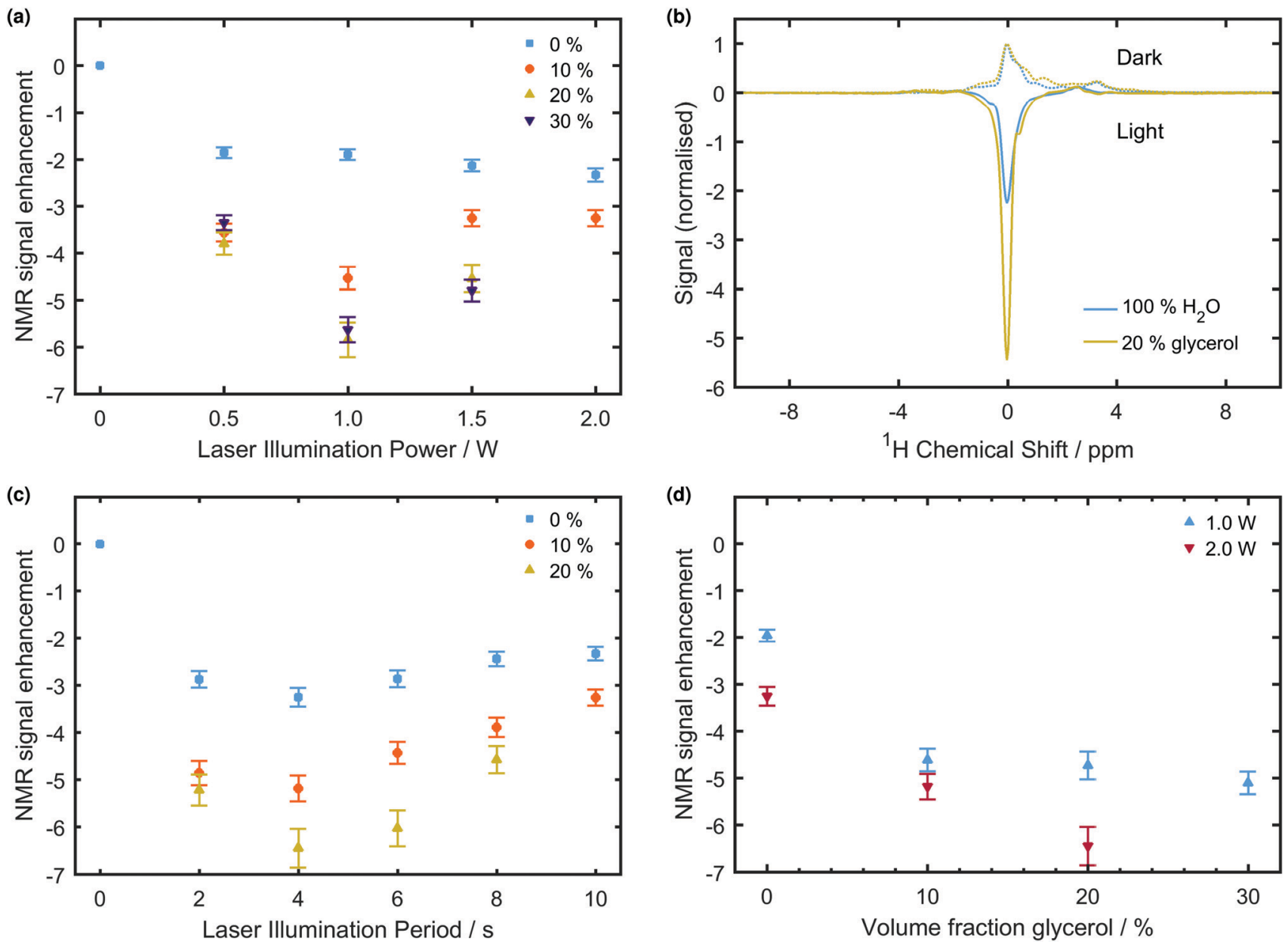

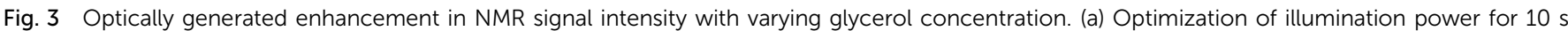

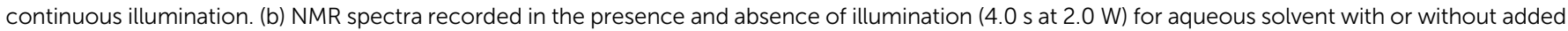

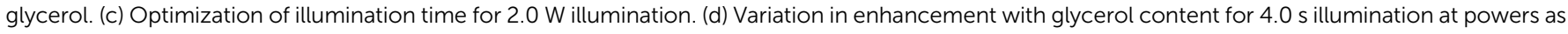

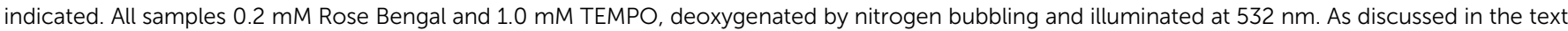

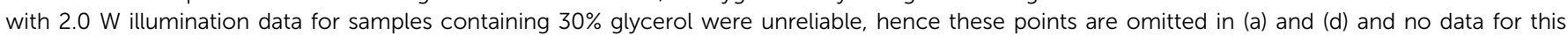
mixture appears in (c). The illumination time dependence for all mixtures with $1.0 \mathrm{~W}$ illumination can be found in Fig. S3 (ESI $\dagger$ ). 
of $0.1 \mathrm{~Hz}$. It can be seen that even for $2 \mathrm{~s}$ illumination $(20 \%$ duty cycle) a significant enhancement is achieved, and such conditions will significantly reduce the likelihood for sample heating and potentially permit recovery of a reversibly photobleached sample. Data for lower illumination power $(1.0 \mathrm{~W})$ also showed significant enhancement with reduced duty cycle, and signs of a plateau for longer illumination times (Fig. S3, ESI $\dagger$ ). The scope for maintaining enhancements using a pulsed laser to produce much lower duty cycle trains of pulses has been discussed elsewhere and remains a target for future experimental investigation. ${ }^{23}$

As noted elsewhere the build-up time for the optical enhancement is expected to be governed by the nuclear relaxation time $T_{1 \mathrm{n}}{ }^{23,37}$ values for which are given in Table 2. While one might therefore expect maximal polarization to be achieved with illumination for $\sim 5 T_{1 \mathrm{n}}$, corresponding to $3.5-7.0 \mathrm{~s}$ in the present case, illumination longer than $4 \mathrm{~s}$ reduced the enhancement obtained. This is likely the result of unwanted heating effects when using a high power illumination source, similar to observations of Liu et al. who noted polarization build up at twice the expected rate. ${ }^{37}$ Laser induced heating was particularly problematic in the most viscous solvent mixture when using the highest illumination power of $2.0 \mathrm{~W}$, with data showing poor reproducibility under these conditions. For this reason only data recorded with illumination powers of up to $1.5 \mathrm{~W}$ are presented for samples containing $30 \%$ glycerol by volume.

To rationalize the variation in NMR enhancement with addition of glycerol, Fig. 3d, it is necessary to introduce the Overhauser formula: ${ }^{38}$

$$
\varepsilon=\frac{\left\langle I_{\mathrm{Z}}\right\rangle-I_{0}}{I_{0}}=-\xi f_{\mathrm{eff}} \frac{\gamma_{\mathrm{e}}}{\gamma_{\mathrm{n}}}
$$

where $\xi$ is the electron-nuclear coupling factor, $f$ the leakage factor, $s_{\text {eff }}$ the effective saturation factor and $\gamma_{\mathrm{e}, \mathrm{n}}$ the electron and nuclear gyromagnetic ratios. As $\left|\gamma_{\mathrm{e}} / \gamma_{\mathrm{H}}\right| \sim 660$, in the dipolar coupled case $(\xi \leq+0.5)$ using thermally polarized electrons the maximum enhancement for protons is $\sim 330$ since the other factors cannot exceed one. Optical polarization however offers scope for the saturation factor $s_{\text {eff }}=\left(S_{0}-\left\langle S_{\mathrm{Z}}\right\rangle\right) / S_{0}$ to take much higher values leading to significantly larger enhancements. $^{3,37}$ It is clear from Fig. 2a that with pulsed illumination the maximum transient value of $s_{\text {eff }}$ can be much

Table 2 Variation in NMR parameters with addition of glycerol to the deoxygenated aqueous solution. Nuclear relaxation times in the presence $\left(T_{1 n}\right)$ and absence $\left(T_{1 n}^{0}\right)$ of TEMPO radical $(1.0 \mathrm{mM})$ were determined by inversion recovery and used to calculate the leakage factor $f$. Quoted uncertainties are from the single exponential fit to the relaxation data. The coupling factor $\xi$ was calculated according to the FFHS method

\begin{tabular}{lllll}
\hline $\begin{array}{l}\text { Volume fraction } \\
\text { glycerol/\% }\end{array}$ & $T_{1 \mathrm{n}}^{0} / \mathrm{s}$ & $T_{1 \mathrm{n}} / \mathrm{s}$ & $f$ & $\xi$ \\
\hline 0 & $2.59 \pm 0.05$ & $1.39 \pm 0.05$ & $0.46 \pm 0.02$ & 0.37 \\
10 & $2.11 \pm 0.06$ & $1.19 \pm 0.03$ & $0.44 \pm 0.02$ & 0.34 \\
20 & $1.72 \pm 0.05$ & $0.96 \pm 0.02$ & $0.44 \pm 0.02$ & 0.30 \\
30 & $1.13 \pm 0.04$ & $0.70 \pm 0.01$ & $0.38 \pm 0.01$ & 0.24
\end{tabular}

greater than unity, but this value in fact reduces as glycerol is added to the solution (Table 1), whereas the NMR enhancement using continuous-wave illumination increases.

Before considering the change in electronic hyperpolarization with glycerol addition the effect on the other parameters in eqn (14) must be accounted for. The leakage factor $f=1-T_{1 \mathrm{n}} / T_{1 \mathrm{n}}^{0}$, where the superscript indicates the relaxation time in the absence of radical, was obtained from ${ }^{1} \mathrm{H}$ NMR inversion recovery data. Both the nuclear relaxation time and leakage factor decrease upon addition of glycerol (Table 2). The coupling factor is also expected to decrease with increasing viscosity, being strongly dependent on the tumbling rate of the molecules. Using the force free hard sphere (FFHS) approximation introduced by Hwang and Freed ${ }^{39}$ it is possible to calculate the spectral density and hence coupling factor, from the correlation time $\tau_{\mathrm{c}}$ of the radical. ${ }^{40,41}$ While the Han group use this approach to determine local water dynamics from enhancement values in microwave-driven Overhauser $\mathrm{DNP},{ }^{41}$ here we estimate the variation in coupling factor upon glycerol addition (Table 2) by using literature values of the closest approach distance and diffusion constants for the radical and water molecules, ${ }^{40,41}$ and scaling these according to the changing solution viscosity. Details of the spectral density function used and discussion of this choice are provided elsewhere. ${ }^{23}$

Considering the leakage and coupling factors both reduce on addition of glycerol it is clear that the increased NMR enhancement must arise from a significantly increased saturation factor. By rearranging eqn (14) we determined the effective saturation factor in optical DNP experiments (Fig. 4a). With 1.0 W illumination the saturation factor increases across the series as the glycerol content is increased, implying that the maximal enhancements recorded in the $20 \%$ glycerol case arise from the interplay of increasing saturation factor with the decreasing leakage and coupling factors. A similar compromise between saturation and leakage factors was previously recognised as determining optimal radical concentrations for the optical DNP method. ${ }^{3}$

Looking at the absolute value of the saturation factor in all cases $s_{\text {eff }}<0.1$, with only a small increase upon doubling the illumination power from $1.0 \mathrm{~W}$ to $2.0 \mathrm{~W}$, hence the optical DNP method does not yet compete with microwave driven methods for which $s_{\text {eff }} \sim 1$ has been achieved. ${ }^{42-45}$ Further optimization of the polarizing system is therefore required, for which it will be useful to quantify the extent of electronic hyperpolarization achieved in different systems. It is notable that the saturation factor for $1.0 \mathrm{~W}$ illumination is higher in the $30 \%$ glycerol case than in the absence of added glycerol, despite the relative electronic polarization per quenching event $P_{\text {net }}$ being within experimental uncertainty identical for these two volume fractions (Table 1). An alternative measure of the efficiency of optical polarization is therefore needed.

\section{Quantifying electronic polarization}

In light of the difficulties discussed above in determining the polarization per quenching event $P_{\text {net }}$ and the fact that alone this value cannot provide the trend in saturation factors under continuous illumination conditions relevant to optical DNP, we 
(a)

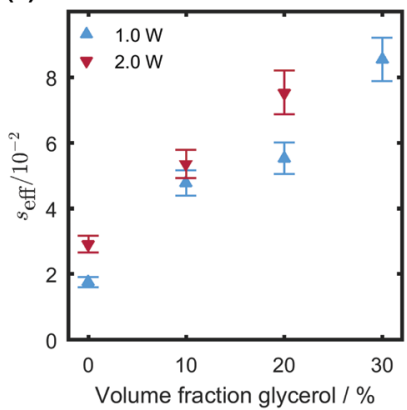

(b)

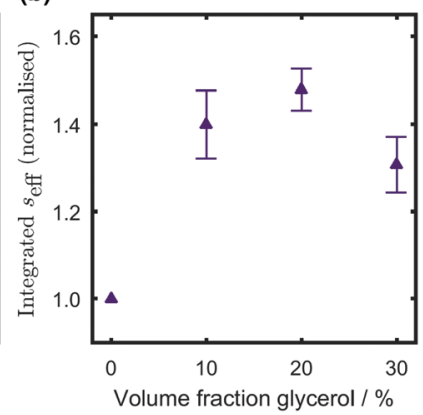

Fig. 4 Variation in $S_{\text {eff }}$ calculated from (a) optical DNP experiments (4 $\mathrm{s}$ illumination period at power as indicated, $1.0 \mathrm{mM}$ TEMPO) and (b) through direct time domain integration of the electronic magnetization in pulsed EPR experiments ( $6 \mathrm{~mJ}$ per pulse, $0.2 \mathrm{mM}$ TEMPO). All samples contain $0.2 \mathrm{mM}$ Rose Bengal and were deoxygenated by nitrogen bubbling prior to measurement.

consider alternative measures of RTPM efficiency. Ideally these should be readily assessed by consideration of directly measurable parameters rather than relying on accurate knowledge of numerous kinetic parameters and quantum yield values. The electronic magnetization $\left\langle S_{\mathrm{Z}}\right\rangle$ may be determined unambiguously and $s_{\text {eff }}(t)$ calculated by reference to the thermal magnetization $S_{0}$ in pulsed EPR experiments, assuming care is taken to remove any instrumental baseline by an appropriate phase cycling (echo detection) or field jumping (FID detection) procedure. This value is directly dependent upon excitation conditions meaning a common standard is required (e.g. the Rose Bengal/TEMPO system used as a reference here) for comparisons between measurements using different experimental arrangements. The maximum achievable value directly indicates the signal enhancement that may be achieved if utilizing electronic hyperpolarization to boost EPR sensitivity, but the peak magnetization (max. $s_{\text {eff }}$, Table 1 ) decreases as glycerol is added to the system whereas the saturation factor relevant to polarization transfer to generate nuclear hyperpolarization has been shown to increase (Fig. 4a). This is as a result of increased persistence time of the electronic magnetization, hence for transfer to nuclei it would be useful to derive a measure of electronic polarization that takes longevity of polarization into account.

The time integral of $s_{\text {eff }}$ obtained from delay after flash EPR measurements is shown in Fig. $4 \mathrm{~b}$ as a function of glycerol content. The value of this integral increases with the volume fraction of glycerol because although the peak magnetization progressively decreases, the polarization is also becoming longer lived. In this case the qualitative trend appears to mirror that in $s_{\text {eff }}$ obtained from optical DNP measurements (Fig. 4a), though there is a discrepancy for the highest glycerol content. The approximation that the viscous solvent mixtures can be treated according to a single bulk diffusion constant in calculating the coupling factor and hence optical DNP saturation factor is a potential source of this discrepancy, and it should be recalled that for this volume fraction the polarization $P_{\text {net }}$ deviated from predicted trends. A quantitative correspondence

between the saturation factors obtained from optical DNP and pulsed EPR studies has not yet been established and these measurements necessarily correspond to different illumination conditions and radical concentrations. It is, however, proposed that as a directly measurable quantity that reflects a combination of both the magnitude and persistence timescale of RTPM generated electronic hyperpolarization, the timeintegrated electronic magnetization is a parameter that should be considered in the search for optimal polarization systems for the future implementation of the optical DNP method.

\section{Future directions}

The ultimate goal of the optical DNP method must be to increase the NMR sensitivity for a substrate of interest rather than the solvent, by which we mean increase for that substrate the signal-to-noise per unit square root of time compared to an optimized non-DNP NMR experiment. ${ }^{46}$ This should be achieved at a sufficiently high magnetic field to provide good chemical shift resolution, typically accepted as a minimum of 14.1 $\mathrm{T}$ for biomolecular NMR applications. The challenge for the optical DNP method is that both the coupling factor $(\xi)$ for dipolar coupled protons, and magnitude of the RTPM generated electronic polarization $\left(\left|P_{\text {net }} / P_{\text {eq }}\right|\right)$, are expected to decrease at higher magnetic fields. Our results for the viscosity dependence of $P_{\text {net }}$ already call in to question the validity of existing numerical models of the RTPM so experimental verification of the predicted magnetic field dependence is warranted, but it is a reasonable hypothesis that efficiency will decrease at higher magnetic fields where the Zeeman splitting of the radical-triplet pair becomes comparable to the exchange interaction. ${ }^{19}$ In this weak exchange limit the LAC region is shifted to separations of less than the van der Waals contact distance making the region inaccessible (Fig. 1). This could be circumvented by polarizing the nuclei at a lower magnetic field before shuttling to a higher field for measurement, an approach already applied to overcome the reduction of coupling factor in microwave pumped Overhauser DNP; a system shuttling from $0.34 \mathrm{~T}$ to $14.09 \mathrm{~T}$ was able to demonstrate DNP enhancements of up to -2.4 on L-tryptophan by microwave pumping a deuterated ${ }^{15} \mathrm{~N}$ nitroxide radical in aqueous solution. ${ }^{45}$ This provides confidence that polarization transfer to solutes can be achieved and unfavourable magnetic field dependencies can be overcome. Given the saturation factor was close to one in this study, the result is close to the theoretical limit for the maximum achievable enhancement in the microwave pumping case $(-7.9$ when the magnetic field ratio is considered) limited only by a coupling factor estimated as 0.23-0.27 and leakage factor of 0.89 . The overall enhancement could be significantly increased if the shuttle approach can be replicated with optical pumping to give an effective saturation factor exceeding one. Another notable feature of this shuttle study is that global enhancement factors of up to -4.2 were also reported, these global values accounting for radical induced line broadening and differences in the repetition rate of optimal NMR and DNP experiments, ${ }^{47}$ to show that shuttle DNP offers a genuine improvement over a non-DNP experiment. 
To increase the effective saturation factor in optical DNP the electronic polarization step must be optimized. Reliance on diffusive encounters between the dye and radical was already identified as a limiting factor, leading the saturation factor to be maximised at low radical concentrations that give a sub-optimal leakage factor. ${ }^{3}$ This may be alleviated by linked dye-radical systems, with development of such polarizers targeted at improved liquid-state optical DNP performance already underway. ${ }^{35,48,49}$ As we have shown such efforts should consider not only the magnitude of the electronic polarization generated but also the persistence timescale of this polarization, as this significantly impacts upon the electronic saturation factor achieved under DNP conditions. At present a ten-fold increase in the saturation factor is needed to allow optical pumping to match the theoretical enhancement limit of microwave driven Overhauser DNP, provided the leakage and coupling factors can be simultaneously maximised to ensure efficient transfer of the optically generated electronic polarization to nuclei. The coupling factor for any new system requires direct measurement as has been demonstrated using the pulsed electron-electron double resonance (ELDOR) method for the fullerene-nitroxide polarizing agents developed by Bennati and co-workers, although ultimately the optical polarization in these systems was low. ${ }^{37,44}$ To simultaneously optimize all of these requirements in a single photo-stable system is undeniably challenging, but reflecting on the significant advances made in rational design of bi-radical polarizing agents for solid-state DNP illustrates what might be achieved through concerted synthetic, spectroscopic and theoretical efforts. ${ }^{50}$

\section{Conclusions}

We have demonstrated the effect of added glycerol on the dynamic electron and nuclear polarization generated by photoexcitation of Rose Bengal/TEMPO, a water soluble system reported to demonstrate one of the largest known RTPM hyperpolarization efficiencies. By optimizing solvent viscosity the magnitude of the nuclear polarization has been increased to nearly six times thermal levels, the highest enhancement yet reported for the burgeoning liquid-state optical-DNP method. This is despite a reduction in the peak electronic magnetization and is attributed to an increase in the effective electronic saturation factor under continuous illumination due to slower electronic relaxation. The electronic polarization generated per triplet quenching event, the conventional measure of RTPM efficiency, is difficult to determine unambiguously and is insufficient to fully describe the changing behaviour of the system upon glycerol addition. We therefore propose time averaged electronic magnetization is adopted as a means of quantifying RTPM efficiency for nuclear polarization applications. While the complexity of the phenomenon and various competing factors prevent rational design of improved dyeradical systems, this metric should form a key part of systematic experimental screening of candidates. ${ }^{37}$

\section{Conflicts of interest}

There are no conflicts of interest to declare.

\section{Acknowledgements}

We thank the Engineering and Physical Sciences Research Council (EPSRC) for financial support (EP/N007875/1, EP/ J500045/1), and Bruker UK Ltd for an equipment loan. CJW thanks the UoW Department of Physics for startup funds enabling the purchase of the pulsed laser source used in this work, and Prof. Christopher Kay for helpful discussions.

\section{References}

1 J.-H. Ardenkjaer-Larsen, G. S. Boebinger, A. Comment, S. Duckett, A. S. Edison, F. Engelke, C. Griesinger, R. G. Griffin, C. Hilty, H. Maeda, G. Parigi, T. Prisner, E. Ravera, J. van Bentum, S. Vega, A. Webb, C. Luchinat, H. Schwalbe and L. Frydman, Angew. Chem., Int. Ed., 2015, 54, 9162-9185.

2 J. van Bentum, B. van Meerten, M. Sharma and A. Kentgens, J. Magn. Reson., 2016, 264, 59-67.

3 M. W. Dale and C. J. Wedge, Chem. Commun., 2016, 52, 13221-13224.

4 C. Corvaja, E. Sartori, A. Toffoletti, F. Formaggio, M. Crisma and C. Toniolo, Biopolymers, 2000, 55, 486-495.

5 C. Corvaja, E. Sartori, A. Toffoletti, F. Formaggio, M. Crisma, C. Toniolo, J.-P. Mazaleyrat and M. Wakselman, Chem. Eur. J., 2000, 6, 2775-2782.

6 E. Sartori, A. Toffoletti, F. Rastrelli, C. Corvaja, A. Bettio, F. Formaggio, S. Oancea and C. Toniolo, J. Phys. Chem. A, 2003, 107, 6905-6912.

7 A. Kawai, S. Mori, K. Tsuji and K. Shibuya, Appl. Magn. Reson., 2010, 38, 205-216.

8 A. Agostini, D. M. Palm, H. Paulsen and D. Carbonera, J. Phys. Chem. Lett., 2018, 9, 672-676.

9 D. J. Cheney and C. J. Wedge, Electron Paramagn. Reson., 2019, 26, 89-129.

10 M. Di Valentin, M. Albertini, E. Zurlo, M. Gobbo and D. Carbonera, J. Am. Chem. Soc., 2014, 136, 6582-6585.

11 M. Di Valentin, M. Albertini, M. G. Dal Farra, E. Zurlo, L. Orian, A. Polimeno, M. Gobbo and D. Carbonera, Chem. Eur. J., 2016, 22, 17204-17214.

12 M. G. Dal Farra, S. Ciuti, M. Gobbo, D. Carbonera and M. Di Valentin, Mol. Phys., 2018, 117, 2673-2687.

13 M. G. Dal Farra, S. Richert, C. Martin, C. Larminie, M. Gobbo, E. Bergantino, C. R. Timmel, A. M. Bowen and M. Di Valentin, ChemPhysChem, 2019, 20, 931-935.

14 K. Tateishi, M. Negoro, S. Nishida, A. Kagawa, Y. Morita and M. Kitagawa, Proc. Natl. Acad. Sci. U. S. A., 2014, 111, 7527-7530.

15 M. Negoro, A. Kagawa, K. Tateishi, Y. Tanaka, T. Yuasa, K. Takahashi and M. Kitagawa, J. Phys. Chem. A, 2018, 122, 4294-4297.

16 A. Blank and H. Levanon, J. Phys. Chem. A, 2001, 105, 4799-4807. 
17 C. Blättler, F. Jent and H. Paul, Chem. Phys. Lett., 1990, 166, 375-380.

18 M. D. E. Forbes, L. E. Jarocha, S. Sim and V. F. Tarasov, Adv. Phys. Org. Chem., 2013, 47, 1-83.

19 A. Kawai and K. Shibuya, J. Photochem. Photobiol., C, 2006, 7, 89-103.

20 H. Takahashi, M. Iwama, N. Akai, K. Shibuya and A. Kawai, Mol. Phys., 2014, 112, 1012-1020.

21 S. S. Kim and S. I. Weissman, Rev. Chem. Intermed., 1979, 3, 107-120.

22 G. H. Goudsmit, H. Paul and A. I. Shushin, J. Phys. Chem., 1993, 97, 13243-13249.

23 D. J. Cheney and C. J. Wedge, J. Chem. Phys., 2020, 152, 034202.

24 L. Ludvíková, P. Friš, D. Heger, P. Šebej, J. Wirz and P. Klán, Phys. Chem. Chem. Phys., 2016, 18, 16266-16273.

25 MATLAB. 9.2.0.556344 (R2017a), The Mathworks Inc., Natick, Massachusetts, 2017.

26 D. C. Neckers, J. Photochem. Photobiol., A, 1989, 47, 1-29.

27 A. W.-H. Mau, O. Johansen and W. H. F. Sasse, Photochem. Photobiol., 1985, 41, 503-509.

28 R. Owenius, G. E. Terry, M. J. Williams, S. S. Eaton and G. R. Eaton, J. Phys. Chem. B, 2004, 108, 9475-9481.

29 J. R. Biller, H. Elajaili, V. Meyer, G. M. Rosen, S. S. Eaton and G. R. Eaton, J. Magn. Reson., 2013, 236, 47-56.

30 A. Leavesley, C. B. Wilson, M. Sherwin and S. Han, Phys. Chem. Chem. Phys., 2018, 20, 9897-9903.

31 K. Murata and H. Tanaka, Nat. Mater., 2012, 11, 436-443.

32 A. Kawai and K. Obi, J. Phys. Chem., 1992, 96, 5701-5704.

33 Y. Kobori, K. Takeda, K. Tsuji, A. Kawai and K. Obi, J. Phys. Chem. A, 1998, 102, 5160-5170.

34 A. I. Shushin, J. Chem. Phys., 1993, 99, 8723-8732.

35 V. Rane and R. Das, J. Phys. Chem. A, 2015, 119, 5515-5523.

36 A. I. Shushin, J. Phys. Chem. A, 2014, 118, 11355-11363.
37 G. Liu, S.-H. Liou, N. Enkin, I. Tkach and M. Bennati, Phys. Chem. Chem. Phys., 2017, 19, 31823-31829.

38 K. H. Hausser and D. Stehlik, Adv. Magn. Reson., 1968, 3, 79-139.

39 L. Hwang and J. H. Freed, J. Chem. Phys., 1975, 63, 118-130. 40 P. J. M. van Bentum, G. H. A. van der Heijden, J. A. Villanueva-Garibay and A. P. M. Kentgens, Phys. Chem. Chem. Phys., 2011, 13, 17831-17840.

41 J. M. Franck, A. Pavlova, J. A. Scott and S. Han, Prog. Nucl. Magn. Reson. Spectrosc., 2013, 74, 33-56.

42 M.-T. Türke and M. Bennati, Phys. Chem. Chem. Phys., 2011, 13, 3630-3633.

43 N. Enkin, G. Liu, I. Tkach and M. Bennati, Phys. Chem. Chem. Phys., 2014, 16, 8795-8800.

44 N. Enkin, G. Liu, M. del Carmen Gimenez-Lopez, K. Porfyrakis, I. Tkach and M. Bennati, Phys. Chem. Chem. Phys., 2015, 17, 11144-11149.

45 P. Lottmann, T. Marquardsen, A. Krahn, A. Tavernier, P. Höfer, M. Bennati, F. Engelke and C. Griesinger, Appl. Magn. Reson., 2012, 43, 207-221.

46 D. Lee, S. Hediger and G. De Paëpe, Solid State Nucl. Magn. Reson., 2015, 66-67, 6-20.

47 V. Vitzthum, F. Borcard, S. Jannin, M. Morin, P. Miéville, M. A. Caporini, A. Sienkiewicz, S. Gerber-Lemaire and G. Bodenhausen, ChemPhysChem, 2011, 12, 2929-2932.

48 A. K. Tripathi, V. Rane, S. Kundu and R. Das, J. Chem. Phys., 2019, 151, 154305.

49 A. Tripathi and V. Rane, J. Phys. Chem. B, 2019, 123, 6830-6841.

50 D. J. Kubicki, G. Casano, M. Schwarzwälder, S. Abel, C. Sauvée, K. Ganesan, M. Yulikov, A. J. Rossini, G. Jeschke, C. Copéret, A. Lesage, P. Tordo, O. Ouari and L. Emsley, Chem. Sci., 2016, 7, 550-558. 